Maximization of regional probabilities using Optimal Surface Graphs application to carotid artery segmentation in MRI

Arias Lorza, Andres M.; Van Engelen, Arna; Petersen, Jens; Van Der Lugt, Aad; De Bruijne, Marleen

Published in:

Medical Physics

DOI:

$10.1002 / \mathrm{mp} .12771$

Publication date:

2018

Document version

Peer reviewed version

Document license:

Unspecified

Citation for published version (APA):

Arias Lorza, A. M., Van Engelen, A., Petersen, J., Van Der Lugt, A., \& De Bruijne, M. (2018). Maximization of regional probabilities using Optimal Surface Graphs: application to carotid artery segmentation in MRI. Medical Physics, 45(3), 1159-1169. https://doi.org/10.1002/mp.12771 


\title{
Maximization of Regional probabilities using Optimal Surface Graphs: Application to Carotid Artery Segmentation in MRI
}

\author{
Andres M. Arias Lorza ${ }^{1}$, Arna van Engelen ${ }^{1}$, Jens Petersen ${ }^{2}$, Aad van der Lugt ${ }^{3}$, Marleen de Bruijne ${ }^{1,2, *}$ \\ ${ }^{1}$ Biomedical Imaging Group Rotterdam, Departments of Radiology and Medical Informatics, Erasmus MC, The Netherlands. \\ ${ }^{2}$ Department of Computer Science, University of Copenhagen, Denmark. \\ ${ }^{3}$ Department of Radiology, Erasmus MC, The Netherlands. * marleen.debruijne@erasmusmc.nl
}

(Dated: February 7, 2018)

\begin{abstract}
Purpose We present a segmentation method that maximizes regional probabilities enclosed by coupled surfaces using an Optimal Surface Graph (OSG) cut approach. This OSG cut determines the globally optimal solution given a graph constructed around an initial surface. While most methods for vessel wall segmentation only use edge information, we show that maximizing regional probabilities using an OSG improves the segmentation results. We applied this to automatically segment the vessel wall of the carotid artery in magnetic resonance images.

Methods First, voxel-wise regional probability maps were obtained using a Support Vector Machine classifier trained on local image features. Then the OSG segments the regions which maximizes the regional probabilities considering smoothness and topological constraints.

Results The method was evaluated on 49 carotid arteries from 30 subjects. The proposed method shows good accuracy with a Dice wall overlap of $74.1 \% \pm 4.3 \%$, and significantly outperforms a published method based on an OSG using only surface information, the obtained segmentations using voxel-wise classification alone, and another published artery wall segmentation method based on a deformable surface model. Intra-class correlations (ICC) with manually measured lumen and wall volumes were similar to those obtained between observers. Finally, we show a good reproducibility of the method with $I C C=0.86$ between the volumes measured in scans repeated within a short time interval.

Conclusions In this work a new segmentation method that uses both an OSG and regional probabilities is presented. The method shows good segmentations of the carotid artery in MRI and outperformed another segmentation method that uses OSG and edge information and the voxelwise segmentation using the probability maps.
\end{abstract}

\section{INTRODUCTION}

Multi-region coupled surface segmentation is an important topic as several applications are represented by coupled surfaces, such as vessel wall segmentation, intra-retinal layers detection, and pulmonary airway wall segmentation. In the specific case of the carotid artery vessel wall, its segmentation is required to make a proper analysis of potential presence and severity of atherosclerotic plaques. Such analysis is important as plaques may rupture resulting in a stroke [1]. Wall segmentation of the carotid artery is possible in MRI, however, manual segmentation is time consuming and subject to inter-observer variability [2].

Several methods to automatically segment the carotid artery vessel wall in MRI have been proposed in the literature [3-6]. In most of these methods, the segmentation is obtained by performing a minimization of a cost function which generally integrates features and geometric vascular models and incorporates smoothness and topological constraints in the segmentation solution. However, lack of proper features, erroneous models, and an inaccurate cost function or optimizer may lead to segmentation errors. All these methods use a limited set of features: image intensity derivatives $[3,5,6]$, or intensity Probability Density Function (PDF) [4]. However, these features are not sufficient to describe the artery wall adequately in all possible cases. Further, optimizers such as the one used by Klooster et al. [3] and Hameeteman et al. [6] may get stuck at a local minimum of the cost function. Therefore, a more descriptive set of features and an optimizer that guarantees a global optimum of a cost function based on those features would be desirable.

Promising results have been obtained using graph-based optimization methods in different applications [5, 710]. Graph-based methods obtain a global minimization of a cost function with low processing times [11]. This cost function is represented in the graph, and the minimization is performed by a graph-cut approach. However, formulating meaningful cost functions represented by the graph can be a challenging problem.

A class of graph-based segmentation methods commonly called Optimal Surface Graph (OSG) is especially interesting. Here the graph is built based on an initial shape, allowing an easy integration of prior shape information in 
the cost function [12]. Previously, we presented a method to segment the carotid artery vessel wall in MRI using an OSG model [5]. The cost function used edge features, based on directional image derivatives, over the inner and outer vessel wall, integrating smoothness and topology constraints. Generally good results were observed, however, segmentation errors may arise as the carotid vessel wall in MRI can have low contrast with the background and/or show high intensity variation, especially in plaque regions, as depicted in figure 1(a). In such cases the edges are not clearly visible and are therefore more difficult to detect using image derivatives as shown in figures 1(b) (inner border) and 1(c) (outer border). In these cases, edge information is not sufficient to obtain good segmentation results, and incorporating information from the regions bounded by the sought surfaces may help to obtain better segmentation. To do this, the segmentation problem has to be reformulated to identify the different bounded regions, instead of finding the surfaces.

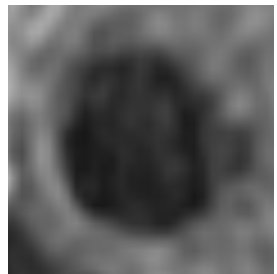

(a)

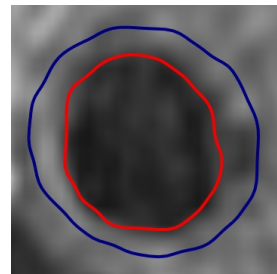

(b)

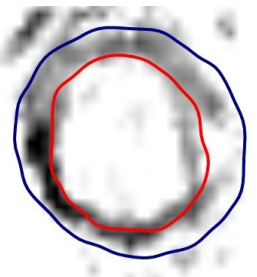

(c)

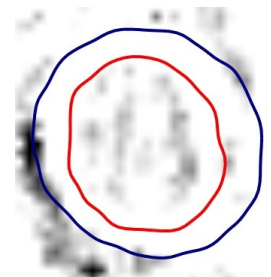

(d)

FIG. 1: Example of an MRI image of the carotid artery (a) with manual annotations overlaying (b) and the local edge information features based on directional image derivatives for the inner vessel wall border (c) and the outer border (d) as described in Arias et al. [5]. Manual annotations for inner and outer border are represented by the red and blue contours respectively.

Multi-region segmentation methods combining global optimization techniques with regional information have been used before [4, 13-16]. Lecellier et al. [16] show an overview of segmentation methods based on maximizing a certain distance between features PDFs on the segmented regions or maximizing the probabilities within the regions. Here, relevant feature PDFs are used, and the optimization is based on evolution of deformable models or convex optimization techniques. Similarly, Ukwatta et al. [4] introduced a global optimization contour evolution method based on matching the intensity PDFs of the carotid artery wall in MRI to the training images PDFs. A convex relaxation of a cost based on this PDFs is minimized using a continuous max-flow model. Lecellier et al. [16] and Ukwatta et al. [4] presented interesting approaches as these can optimize the cost without a graph, thus avoiding graph discretization errors. However, as these approaches require an initial estimation of the PDFs, errors may arise in case of inaccuracies in this estimation. Better results may be obtained if more regional features are used besides intensity, and if an explicit shape prior is integrated in the model.

The use of graph models together with regional information has become popular in image segmentation. For instance, Delong and Boykov [13] proposed a voxel-wise graph model which incorporates the spatial distribution of colors within an object to segment multiple enclosed region structures. In contrast, OSG as opposed to voxel-wise graph models are built based on an initial shape. This may be beneficial to segment structures with complex geometry. Wu et al. [14], Sun et al.[17], and Haeker et al. [15] used a similar OSG based on Li et al. [18] graph model. The OSG model presented in $\mathrm{Li}$ et al.[18] unfolds the image using an initialization, transforming the problem to a terrain-like surface segmentation. Then the optimization problem is formulated to find a closed set of vertices with minimum cost. Wu et al.[14] used this graph model to minimize the intraclass intensity variance, Sun et al.[17] used it toguether with regional terms based on intensity PDFs to segment coronary arteries, and Haeker et al.[15] used it to maximize the regional memberships within a region using intensity based membership functions. However, this unfolding process is not suitable for more complex shapes such as a bifurcating artery. To overcome this problem, Liu et al.[19] transform the model presented by Li et al.[18] by constructing the graph based on normal directed trajectories on the initialization, where the length of the trajectories are limited by the medial axes of the initialization. Similarly, but more suitable for high curvature regions Petersen et al.[10], construct the graph based on smooth flow lines.

We demonstrated in Arias et al. [5] that an OSG model similar to Petersen et al.[10] was suitable to segment the carotid artery bifurcation in MRI. However, due to the errors observed at the outer artery wall when using only directional derivatives as segmentation feature, in this work we incorporate regional information in the OSG aiming to improve the overall segmentation results. We apply this method to segment the carotid artery wall in multi-spectral MRI. We follow a similar approach to incorporate the regional information as in Haeker et al. [15]. The regional information is included in the graph as edge costs, and this cost is defined such that a minimal cut maximizes the enclosed sum of regional probabilities considering smoothness and topological constraints. However, in our model we cannot directly apply the edge cost presented by Haeker et al. [15] as these graph structures are different. While they 
search for the minimum-cost closed set of vertices in a graph $G$ by transforming $G$ to a graph $G_{s t}$ where all vertices are either connected to the source $s$ or sink $t$ of the graph, as in Petersen et al. [10] we do not apply this and instead we specify the problem directly in terms of minimum cost surfaces. Therefore, we reformulated the cost function defined in Haeker et al. [15] such that it can be applied with our graph model. Then the main contribution of this paper is to adapt the graph model presented in Petersen et al.[10] to incorporate regional information in the graph, so after the optimization the enclosed regions that maximize the regional probabilities are obtained. This cost function definition could be used with any OSG model based on Ishikawa [20] graph model such as Petersen et al.[10]. We experimentaly show large improvements of the presented approach over both Arias et al. [5] and a voxelwise segmentation approach using the regional information only. We represent the regional information as Regional Probability Maps (RPMs) obtained using a support vector machine classifier. These RPMs allow us to use multiple descriptive image features from the different MRI sequences, allowing the combination of their complementary information [21], and resulting in a better representation of the regions and more robust segmentation results.

\section{METHOD}

\section{A. Optimal Surface Graph Method}

OSG methods as proposed by Wu et al.[12] allow optimal multiple surface segmentation solutions incorporating topology constraints and shape prior information. In the OSG methods the optimization is defined as the minimization of a cost function represented as the sum of graph edge costs, and the minimization is computed using a minimum cut algorithm. The graph $G=(V, E)$ is composed of vertices $V$ and edges $E$. $V$ is composed of the vertices associated with positions in the image, and the vertices $s$ and $t$ which denote the source and sink points of the graph. The edges $E$ connect the vertices of the graph, and represent the association between vertices. Therefore, to obtain meaningful segmentations, costly edges are expected to connect vertices from the same region, whereas low-cost edges are expected to connect vertices from different regions. The segmentation solution is defined by the minimum cut that separates the graph in two parts: source part $V_{s} \subseteq V$ and sink part $V_{t} \subseteq V$, such that $V_{t}=V \backslash V_{s}, s \in V_{s}, t \in V_{t}$. Given the edge cost $\omega: E \rightarrow \mathbb{R}$, the minimum cut minimizes the total cost of the edges that are being cut represented by $\min _{\left(v_{i} \in V_{s}, v_{j} \in V_{t}\right)} \sum \omega\left(v_{i} \rightarrow v_{j}\right)$ [20]. For multiple surface segmentation, all surfaces are segmented simultaneously using a single minimum cut. Each surface is thus represented by a subgraph and the individual surface subgraphs are coupled using surface coupling edges between vertices of each subgraph. This coupling also allows to incorporate topological constraints in the segmentation solution $[5,10]$.

We follow a similar graph construction approach as presented by Arias et al. [5] to segment the carotid artery wall on MRI. In Arias et al. [5], the graph is constructed starting from a coarse initial segmentation of the innermost surface, and the vertices are grouped by smooth non-intersecting graph columns tracing inwards and outwards from this initial segmentation. Non-intersecting graph columns are guaranteed if these are defined on the trajectory of flow lines of the Gaussian smoothed initial segmentation. Given the flow lines $\boldsymbol{f}_{i}: \mathbb{Z} \rightarrow \mathbb{R}^{3}$ where $i \in\{1, \ldots, N\}$ with $N$ the number of voxels on the initial segmentation surface, the graph vertex $v_{i, k}^{m} \in V$ is associated with the image position $\boldsymbol{f}_{i}(k)$ and a possible position of surface $m \in\{1, \ldots, M\}$, where $M$ is the maximum number of coupled surfaces to segment. The graph columns are defined by the set of vertices $V_{i}^{m}=\left\{v_{i, k}^{m} \mid k \subset \mathbb{Z}, I_{i}, O_{i} \in \mathbb{Z}, k=-I_{i},-I_{i}+1, \ldots, O_{i}-1, O_{i}\right\}$ where the uniform separation distance between vertices is given by $\delta=\left\|\boldsymbol{f}_{i}(k+1)-\boldsymbol{f}_{i}(k)\right\|$. Here $v_{i,-I_{i}}^{m}$ and $v_{i, O_{i}}^{m}$ represent the innermost and outermost vertices of column $V_{i}^{m}$. Several edges are defined in the graph [5]: 1) the edges $v_{i, k}^{m} \rightarrow v_{i, k+1}^{m}$ in the same graph column indicate the relation between inside and outside of surface $m$ such that $\omega\left(v_{i, k}^{m} \rightarrow v_{i, k+1}^{m}\right)$ should be a minimum at the position of the surface; 2) the smoothing penalty edges between neighboring graph columns given by $\left.v_{i, k}^{m} \stackrel{p^{m}}{\longleftrightarrow} v_{j, k}^{m} ; 3\right)$ topological constraint edges in the same graph columns $v_{i, k+1}^{m} \stackrel{\infty}{\rightarrow} v_{i, k}^{m}$; and finally the graph coupling edges $v_{i, k}^{m} \stackrel{\infty}{\rightarrow} v_{i, k+\Delta}^{m+1}$ to guarantee that surface $m+1$ is outside surface $m$ with a minimum distance of $\Delta$ vertices in between; 4) finally, the source vertex $s$ is connected to all innermost vertices, and the sink vertex $t$ to all outermost vertices.

In Arias et al. [5], to get a minimum of $\omega\left(v_{i, k}^{m} \rightarrow v_{i, k+1}^{m}\right)$ at the position of surface $m$, the method favors positions with high image gradient, such that $\omega$ is inversely proportional to the positive or negative part of the first order derivative of the image intensity $I: \mathbb{R}^{3} \rightarrow \mathbb{R}$ along the graph column trajectory $\boldsymbol{f}_{i}$. This is defined by: $\omega\left(v_{i, k}^{m} \rightarrow v_{i, k+1}^{m}\right) \propto$ $\left|\left(\frac{\partial I\left(\boldsymbol{f}_{i}(k)\right)}{\partial k}\right)^{\{+,-\}}\right|^{-1}$, where $I\left(\boldsymbol{f}_{i}(k)\right)$ is the image intensity at the position $\boldsymbol{f}_{i}(k)$. In case the border is defined by a dark to bright contrast (as for the inner wall border of the carotid artery in MRI) then the positive part of the derivative is taken and negative otherwise. An example of these edge costs mapped in the image space are depicted in Figure 1. 


\section{B. Regional Information and OSG}

Ideally, the cost functions as described in Arias et al. [5] would yield low costs at the surface positions. However, in the carotid vessel wall in MRI, the plaque sections and surrounding structures of the vessel are highly variable in intensities, which leads to poor contrast at the border or may even cause other structures to have stronger edges and therefore lower costs than the true vessel borders, as shown in Figure 1. Although cost errors in individual graph columns may be corrected thanks to the smoothing penalty edges and the coupling edges during the global graph minimization, inaccuracies in the final segmentation may still arise.

In these cases where the vessel border is not clearly visible in the image, edge information is not sufficient to get good segmentation results, and better segmentations are expected by using regional information that integrates several features. Let the probability that an image position belongs to region $R_{m}$ be defined by $P_{R_{m}}: \mathbb{R}^{3} \rightarrow[0,1]$. The segmentation problem can be defined as the separation of regions that maximizes the total sum of regional probabilities. Following [15], $R=\bigcup_{m=0, . . M} R_{m}$ is the region to segment, where $R_{0}, \ldots, R_{m}$ are enclosed by the surface $m+1$, and there is no overlap between regions, so $R_{a} \cap R_{b}=\varnothing$ for $a \neq b$ and $a, b \in\{0, \ldots, M\}$, then the optimal segmentation solution is represented by the separation of the regions $R_{0}, \ldots, R_{M}$ that maximizes the total sum of regional probabilities, that is:

$$
\underset{R_{0}, \ldots, R_{M}}{\arg \max }\left(\sum_{m=0}^{M}\left(\sum_{\boldsymbol{x} \in R_{m}} P_{R_{m}}(\boldsymbol{x})\right)\right)
$$

As in Haeker et al.[15], Eq. 1 is modified to get a separate expression for each of the enclosed regions so this can be solved using a graph cut. As the sum of all probabilities in $R$ is a constant: $\sum_{\boldsymbol{x} \in R} P_{R_{m}}(\boldsymbol{x})=$ Constant, and the set of regions that maximizes Eq. 1 is not changed by adding constant values, Eq. 1 is reformulated as:

$$
\underset{R_{0}, \ldots, R_{M}}{\arg \max }\left(\sum_{m=0}^{M}\left(\sum_{\boldsymbol{x} \in R_{m}} P_{R_{m}}(\boldsymbol{x})\right)+\sum_{m=1}^{M-1}\left(\sum_{\boldsymbol{x} \in R} P_{R_{m}}(\boldsymbol{x})\right)\right) .
$$

After expanding and reorganizing Eq. 2, we obtain:

$$
\underset{R_{0}, \ldots, R_{M}}{\arg \max }\left(\sum_{m=0}^{M-1}\left(\sum_{\boldsymbol{x} \in\left\{R_{0}, . ., R_{m}\right\}} P_{R_{m}}(\boldsymbol{x})+\sum_{\boldsymbol{x} \in\left\{R_{m+1}, \ldots, R_{M}\right\}} P_{R_{m+1}}(\boldsymbol{x})\right)\right) .
$$

Eq. 3 defines a maximization based on a sum for each of the enclosed regions in the surfaces. We have to reformulate this equation so it can be solved using a minimum cut in our graph structure. As $R$ can also be represented by the union of the vertex positions in the graph columns, we have that $R=\bigcup_{i=1, \ldots N} f_{i}$. Therefore, replacing each position $\boldsymbol{x} \in R$ by the position in the graph column $\boldsymbol{f}_{i}$, we can further expand Eq. 3 to:

$$
\underset{R_{0}, \ldots, R_{M}}{\arg \max }\left(\sum_{m=0}^{M-1} \sum_{i=1}^{N}\left(\sum_{\substack{f_{i}\left(k_{1}\right) \in R_{0}, . ., R_{m} \\ k_{1} \in-I_{i}, \ldots, O_{i}}} P_{R_{m}}\left(\boldsymbol{f}_{i}\left(k_{1}\right)\right)+\sum_{\substack{f_{i}\left(k_{2}\right) \in R_{m+1}, . ., R_{M} \\ k_{2} \in-I_{i}, \ldots, O_{i}}} P_{R_{m+1}}\left(\boldsymbol{f}_{i}\left(k_{2}\right)\right)\right)\right) .
$$

As the graph columns progressively cross each region from $R_{0}$ to $R_{M}$, for each graph column there is a vertex position $\boldsymbol{f}_{i}\left(k_{i, m}\right)$ given $k_{i, m} \in\left\{-I_{i},-I_{i}+1, \ldots, O_{i}-1, O_{i}\right\}$ located at the outer surface of $R_{m}$ that satisfies that all vertices in the column below and including it are inside the enclosed regions by this surface, that is: $\left\{\boldsymbol{f}_{i}\left(-I_{i}\right), \boldsymbol{f}_{i}\left(-I_{i}+\right.\right.$ $\left.1), \ldots, \boldsymbol{f}_{i}\left(k_{i, m}\right)\right\} \in\left\{R_{0}, . ., R_{m}\right\}$ and $\left\{\boldsymbol{f}_{i}\left(k_{i, m}+1\right), \ldots, \boldsymbol{f}_{i}\left(O_{i}\right)\right\} \in\left\{R_{m+1}, . ., R_{M}\right\}$. Finally, we transform Eq. 4 from a maximization into a minimization that can be solved by computing a minimum graph cut, by introducing the arbitrary constant $K$. Therefore, we can reformulate Eq. 4 to search for these vertex positions instead of the regions:

$$
\underset{k_{i, m}}{\arg \min }\left(\sum_{m=0}^{M-1} \sum_{i=1}^{N}\left(K-\left(\sum_{k_{1}=-I_{i}}^{k_{i, m}} P_{R_{m}}\left(\boldsymbol{f}_{i}\left(k_{1}\right)\right)+\sum_{k_{2}=k_{i, m}+1}^{O_{i}} P_{R_{m+1}}\left(\boldsymbol{f}_{i}\left(k_{2}\right)\right)\right)\right),\right.
$$

Eq. 5 can now be solved using a minimum cut. Given that each graph column is cut only once, we can define 
$\omega\left(v_{i, k}^{m} \rightarrow v_{i, k+1}^{m}\right)$ as:

$$
\omega\left(v_{i, k}^{m} \rightarrow v_{i, k+1}^{m}\right)=K-\left(\sum_{n=-I_{i}}^{k} P_{R_{m}}\left(\boldsymbol{f}_{i}(n)\right)+\sum_{l=k+1}^{O_{i}} P_{R_{m+1}}\left(\boldsymbol{f}_{i}(l)\right)\right) .
$$

Therefore obtaining a minimum cut using the edge costs defined by Eq. 6 guarantees an optimal separation of the regions as defined in Eq. 1.

\section{Regional Probability Maps}

We represent the regional information as a Regional Probability Map (RPM). Accurate RPMs that make use of a large set of image features can be obtained using voxel classification methods. To compute the RPMs, we used a Support Vector Machine (SVM) with a radial basis function kernel. This is a relatively flexible classifier that has proven to be successful in many applications. We performed multi-class classification by combining one-vs-one classifiers. To obtain the RPMs, first pairwise class probabilities are obtained based on the distance to the one-vs-one decision boundary [22]. Subsequently, the pairwise probabilities are combined (as in Wu et al. [23]) to obtain the regional probabilities. Finally, these are normalized to sum to one: $\sum_{t=0}^{M} P_{R_{t}}(\boldsymbol{x})=1$.

\section{EXPERIMENTS AND RESULTS}

We applied the presented coupled OSG approach that maximizes the enclosed regional probabilities to segment the carotid artery bifurcation wall in MRI. We compared the segmentations using the presented method to the segmentations obtained using only edge information as in Arias et al. [5], and to the solution by voxelwise SVM classification inside a Region Of Interest (ROI).

\section{A. Image Data and preprocessing}

We used MRI of the carotid bifurcation from subjects with diseased arteries (stenosis between 30\%-50\%) from the Rotterdam study [21]. The method was evaluated on carotid arteries from 30 subjects where manual annotations were available. From the 60 available arteries, we had to discard 5 because of missing or erroneous manual annotations. Further, arteries from 36 patients who were imaged twice within a short time period were used to assess scan-rescan reproducibility.

Several MRI sequences were acquired: Proton Density Weighted (PD) Black-Blood MRI (BB), PD Echo Planar Imaging MRI (EPI), 3D T1-weighted gradient echo MRI (T1), T2-weighted EPI MRI (T2), and Phase Contrast MRI $(\mathrm{PC})$. The image resolutions are (in-plane voxel size $\times$ Slice thickness): $0.507 \times 0.507 \times 0.9 \mathrm{~mm}$ for BB, $0.703 \times 0.703 \times$ $1 \mathrm{~mm}$ for $\mathrm{PC}, 0.507 \times 0.507 \times 1.2 \mathrm{~mm}$ for $\mathrm{EPI}, 0.703 \times 0.703 \times 0.5 \mathrm{~mm}$ for $\mathrm{T} 1$, and $0.507 \times 0.507 \times 1.2 \mathrm{~mm}$ for $\mathrm{T} 2$. Each sequence provides different information to describe the three regions to segment: lumen, wall, and background. Whereas the PD sequences (BB and EPI) are more commonly used to depict the vessel wall, PC is good to visualize the lumen, and T1 and T2 provide more information on plaque composition in case of disease [21].

The coarse initial segmentation of the innermost surface required to initialize the OSG method was obtained by applying a morphological dilation (using a disk structuring element with radius of $2.5 \mathrm{~mm}$ ) to the vessel lumen centerline, which was obtained by a semi-automatic centerline extraction method using both BB and PC based on minimum shortest cost path presented by Tang et al. [24]. This method requires three marked seed points per artery. Here $\mathrm{PC}$ is rigidly registered to $\mathrm{BB}$, and therefore the initial segmentation is in $\mathrm{BB}$ coordinates, resulting in an OSG also in the same coordinate system.

To all image sequences a N4 bias field correction using the default set of parameters [25] was applied. Subsequently, the four other sequences were non-rigidly registered to BB using the registration configuration presented in Klooster et al. [26]. Subsequently, we applied a linear intensity normalization to the images such that the $5 \%$ intensity percentile $I_{5 \%}$ and $95 \%$ intensity percentile $I_{95 \%}$ were scaled between constant values $\bar{I}_{5 \%}$ and $\bar{I}_{95 \%}$. $\bar{I}_{5 \%}$ and $\bar{I}_{95 \%}$ were chosen such that the highest intensity resolution was achieved while preventing data overflow or underflow. Finally, the images were automatically cropped $22.5 \mathrm{~mm}$ below and $6.3 \mathrm{~mm}$ above the bifurcation point of the carotid artery. A smaller region was used if the marked seed points were defined under this range.

Manually segmented volumes for all 55 arteries were obtained from annotated contours by a radiologist with several years of experience (observer 1) on the BB images using a similar segmentation framework as described in Hameeteman 
et al. [2]. These manual volumes were used to optimize the parameters and evaluate the methods in cross-validation experiments.

\section{B. Experimental setup}

We performed a three-fold cross-validation to train the SVM classifier and to optimize the parameters of the OSG methods. Here three times both classifier training and determining the graph parameters were performed on a training set consisting of two folds of in total 20-21 subjects, and performance was subsequently evaluated on a test set on the held out fold of 10-11 subjects. For the training we used as ground truth the annotations from observer 1. We compared the following methods: OSG method + BB regional probabilities (OSG-BB), OSG method + BB \& EPI regional probabilities (OSG-BBEPI), OSG method + All images regional probabilities (OSG-ALL), OSG using only edge information from BB as in Arias et al. [5] (OSG-Edge), Voxel classification using BB (SVM-BB), Voxel classification using from BB \& EPI (SVM-BBEPI), Voxel classification using using all sequences (SVM-ALL).

As $\mathrm{BB}$ has been reported to be the best sequence to detect the artery wall [21], we chose to use it on its own in OSG-BB, SVM-BB and OSG-Edge. Additionally, as we previously reported that BB and EPI combined gives better or similar results than using only BB [5], we also evaluate those two combined in OSG-BBEPI and SVM-BBEPI. Finally, we used all sequences in OSG-ALL and SVM-ALL to evaluate the combined contribution of all the available sequences.

The training consisted of two steps: firstly we optimized the SVM parameters to obtain the RPMs on each training set, and secondly we optimized the parameters of the OSG methods using these RPMs.

\section{Feature set}

To compute the RPMs, we extracted features from all five image sequences. As described above, RPMs were computed using three sets of features: from BB alone, from BB and EPI, and from all image sequences. Per image, we extracted a set of intensity and derivative features, as these are relatively standard features in image segmentation. Additionally, we obtained position features as we think these are descriptive of the region the voxel belongs. Specifically, we extracted a set of 16 image features per image (so 80 in total for all 5 images), and 3 spatial features:

1) The image intensity;

2-4) Smoothed image intensities using a 3D Gaussian kernel at three different scales: 1, 2 and 3 times the in-plane voxel size $(0.507 \mathrm{~mm})$;

5-10) The 1st order Gaussian directional derivatives at the same three scales, in radially outward direction from the two obtained artery centerlines;

11-16) The 2nd order Gaussian directional derivatives at the same three scales, in radially outward direction from the two centerlines separately;

17,18) The in-plane Euclidean distance to both centerlines separately;

19) The slice position along the centerline with respect to the bifurcation (negative proximal to the bifurcation and positive distal to the bifurcation).

In each individual experiment the features were normalized by scaling each feature in the training set to zero mean and unit standard deviation. The same transformation (X-mean(Ftrain)) / std(Ftrain), with X the feature value in the testset and Ftrain the set of feature values in the trainset) was then applied to this experiment's test data.

\section{SVM classification}

An SVM classifier was trained on the feature vectors of the training set and applied to obtain RPMs in the test images. We used the LIB-SVM toolbox [22] to perform the SVM classification. An example of images and the obtained RPMs are shown in Figure 2.

To train the SVM classifier we sampled training data from all arteries in the train set. For each artery we randomly selected the same number of samples for each of the three regions using the manually segmented volumes: $5 \%$ of all voxels from the smallest region (lumen or wall depending on the vessel), and the same number of voxels randomly taken from the other two regions. Background samples were taken from a region within six voxels around the outer wall border. 


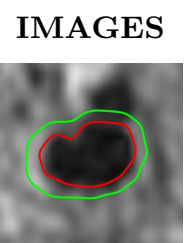

BB

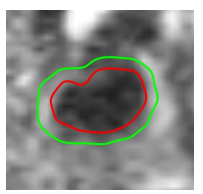

EPI

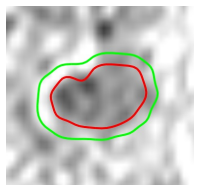

T1

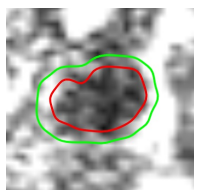

$\mathrm{T} 2$

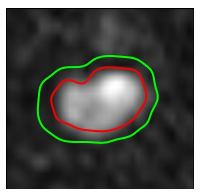

$\mathrm{PC}$
BB

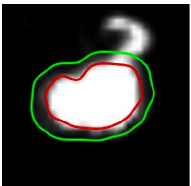

$P_{R_{0}}$

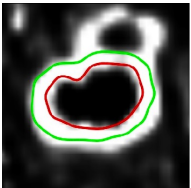

$P_{R_{1}}$

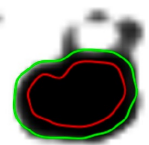

$P_{R_{2}}$

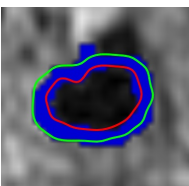

OSG-BB

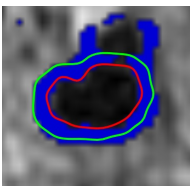

SVM-BB
BB+EPI

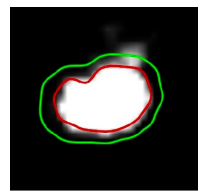

$P_{R_{0}}$

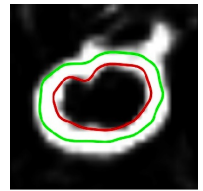

$P_{R_{1}}$

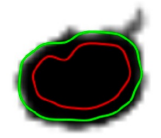

$P_{R_{2}}$

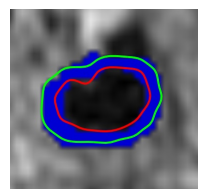

OSG-BBEPI

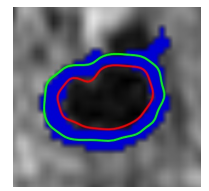

SVM-BBEPI
ALL

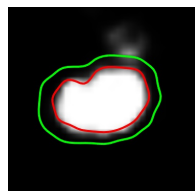

$P_{R_{0}}$

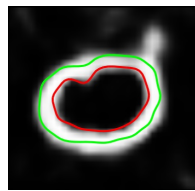

$P_{R_{1}}$

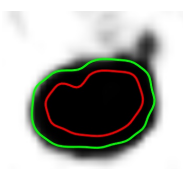

$P_{R_{2}}$

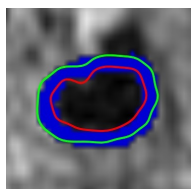

OSG-ALL5

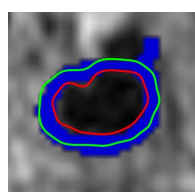

SVM-ALL5

FIG. 2: Image sequences, RPMs and automatic segmentation examples. First column: image sequences after N4 bias field correction, registration, and intensity normalization. Second column: RPMs of lumen, wall, and background respectively, and the wall automatic segmentations using only BB. Third column: RPMs and segmentations using BB and EPI. Fourth column: RPMs and segmentations using all image sequences. The manually annotated inner borders are represented by red, and the outer borders by green contours. The automatic segmented walls are represented in blue.

In our parameter optimization experiments we varied the slack parameter C of the SVM between $\{0.001,0.01, \ldots, 1000\}$, and the SVM kernel radius $\gamma$ between $\{0.0001,0.001, \ldots, 100\}$.

To optimize these parameters we calculated the classification accuracy for each combination of $\mathrm{C}$ and $\gamma$ for each patient using leave-one-patient-out cross-validation within the training set. Per training set the $\mathrm{C}$ and $\gamma$ yielding the highest average accuracy were selected shown in Table I, and an SVM classifier trained on the full training set using the selected $\mathrm{C}$ and $\gamma$ was applied to generate RPMs for the images in the test set.

Additionally, RPMs for the training images were generated using the selected $\mathrm{C}$ and $\gamma$ in a leave-one-patient-out fashion within the training set. These RPMs were used to tune the OSG parameters as described in Section IIIE.

\section{E. Graph Parameter Optimization}

Using the obtained RPMs in the training images, the parameters to optimize in the graph methods are: the standard deviation $\sigma$ to smooth the initial segmentation; the sampling interval $\delta$, the smoothing penalization constants $p^{1}$ and $p^{2}$, and the minimum vertex distance between borders $\Delta$. As in Arias et al. [5], $\delta$ was fixed to $0.35 \mathrm{~mm}$ to generate a higher resolution graph than the original images. As the minimum carotid wall thickness is about $0.8 \mathrm{~mm}$ [27], we fixed $\Delta=2$ vertices, which represents a distance of $0.7 \mathrm{~mm}$ between vertices. We performed a grid search to find the optimal values which optimize the mean Dice Similarity Coefficient (DSC) of the segmented wall compared to 
the manually segmented volumes on each training set. For the OSG using the RPMs, we searched for $p^{1}$ between $\{0,100, \ldots, 500\}, p^{2}$ between $\{0,100, \ldots, 1000\}$, and $\sigma$ between $\{1,1.1, \ldots, 1.5\}$, while for OSG-Edge we searched for $p^{1}$ between $\{0,100, \ldots, 2000\}, p^{2}$ between $\{0,100, \ldots, 2000\}$, and $\sigma$ between $\{0.8,0.9, \ldots, 1.2\}$. The obtained optimal parameters for each method in each training set are shown in table I. In most cases, a similar set of parameters was obtained in all three sets for the different methods. This reflects the robustness of the methods using different data sets.

TABLE I: Optimal parameters for each segmentation method on each training set.

\begin{tabular}{lrrrrrr} 
Method & Train set & $C$ & $\gamma$ & $p^{1}$ & $p^{2}$ & $\sigma$ \\
\hline \multirow{3}{*}{ OSG-BB } & 1 & 100 & 0.01 & 100 & 400 & 1.2 \\
& 2 & 100 & 0.01 & 100 & 500 & 1.1 \\
& 3 & 100 & 0.01 & 300 & 600 & 1.2 \\
OSG-BBEPI & & & & & & \\
& 1 & 10 & 0.01 & 0 & 500 & 1.2 \\
& 2 & 10 & 0.01 & 0 & 400 & 1.1 \\
OSG-ALL & 3 & 10 & 0.01 & 100 & 400 & 1.2 \\
& 1 & 1 & 0.01 & 200 & 200 & 1.2 \\
& 2 & 100 & 0.001 & 200 & 200 & 1.0 \\
& 3 & 1 & 0.01 & 100 & 500 & 1.2 \\
OSG-Edge & & & & & & \\
& 1 & - & - & 900 & 700 & 0.9 \\
& 2 & - & - & 800 & 700 & 0.9 \\
& 3 & - & - & 900 & 700 & 0.9
\end{tabular}

\section{F. Methods Comparison Results}

Using the optimal parameters determined in the training sets, we applied the segmentation method in the held out subjects for testing for all three folds. For this experiment we focus in the comparison of the segmentation methods only and therefore we decided to discard six other arteries with failed centerlines outside the artery.

An example of the image sequences, regional probabilities, and cross-sectional segmentation results is shown for one vessel cross-section in Figure 2. Additional segmentation results are shown in Figure 3. Better wall segmentations using the presented method that combines the OSG and the regional probabilities are observed. The segmentations using the SVM classifier look more irregular and may include neighboring structures in the segmentation. However, when including more image features, the regional probabilities and the SVM-based segmentations improve. The segmentation using OSG-Edge tends to find the stronger edge, which may not be the artery edge and leads to clear errors in examples 1, 3 and 4 in Figure 3. Further, examples 2-6 show a plaque, where the presented method using the RPMs with the OSG obtains fairly good segmentations, indicating that the method performs well in presence of artery disease.

We computed the artery wall, lumen, and complete vessel DSCs for all the 49 arteries using the presented methods. For the segmentations using the SVM only, a ROI of $1.57 \mathrm{~cm}$ radius around the computed centerline was used. This radius was selected because it was the minimum necessary to cover all manually annotated arteries. The DSCs for the different methods are shown in Table II. We performed Friedman analysis with a post-hoc analysis based on Tukey-Kramer testing for multiple comparisons to determine significant differences based on the statistical test (also shown in Table II). The presented methods that maximize the regional probabilities using the OSG are significantly better than the segmentations based on the classifier alone and OSG-Edge $(p<0.05)$. For the SVM segmentations when increasing the number of features (more image sequences) higher average DSC was obtained. The method OSG-Edge performed better than the SVM segmentations. 


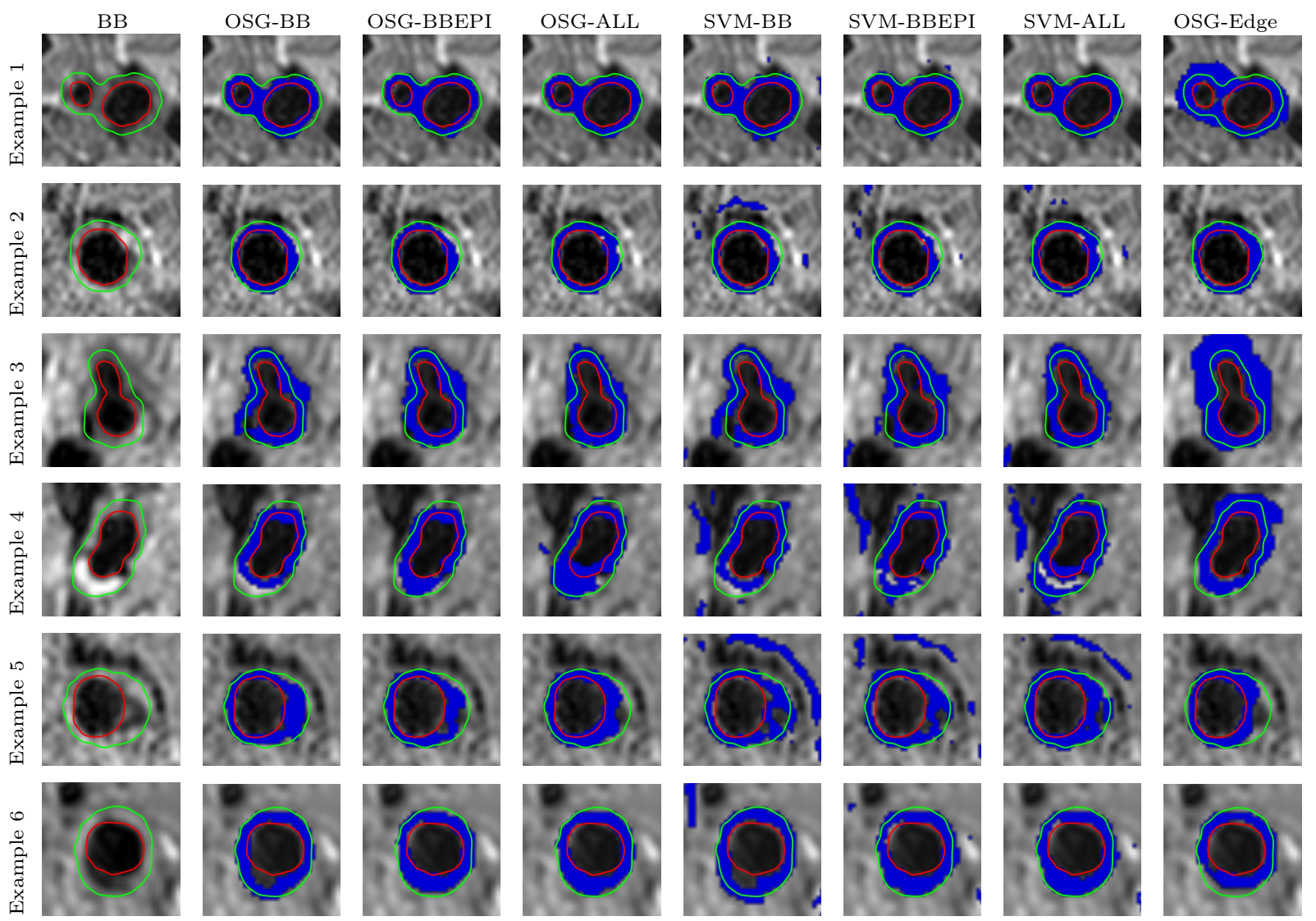

FIG. 3: Examples of segmentation results (for the SVM methods only results inside the ROI are shown). The manually annotated inner borders are represented by red, and the outer borders by green contours. The automatic segmented walls are represented by blue surfaces overlaying the $\mathrm{BB}$ image.

TABLE II: DSC performance for all methods using a data set of 49 arteries, compared with manual annotations from Obs. 1. Values in the same row with the same symbol are not significantly different $(p>0.05)$.

\begin{tabular}{|c|c|c|c|c|c|c|c|}
\hline & OSG-BB & OSG-BBEPI & OSG-ALL & SVM-BB & SVM-BBEPI & SVM-ALL & OSG-Edge \\
\hline Wall DSC & $74.1 \% \pm 4.3 \%^{\dagger}$ & $73.7 \% \pm 5.7 \%^{\dagger}$ & $73.9 \% \pm 5.9 \%^{\dagger}$ & $60.9 \% \pm 6.0 \%^{+}$ & $61.4 \% \pm 8.3 \%^{*+}$ & $65.6 \% \pm 9.1 \%^{\times}$ & $67.5 \% \pm 6.7 \%^{* \times}$ \\
\hline Lumen DSC & $88.3 \% \pm 3.5 \%^{\times}$ & $88.6 \% \pm 3.1 \%^{\times}$ & $88.7 \% \pm 3.8 \%^{\times}$ & $87.6 \% \pm 4.0 \%^{\times}$ & $87.2 \% \pm 5.0 \%^{\times}$ & $86.6 \% \pm 6.0 \%^{\times}$ & $91.9 \% \pm 2.6 \%^{\dagger}$ \\
\hline Complete DSC & $92.7 \% \pm 1.8 \%^{\dagger}$ & $92.6 \% \pm 2.2 \%^{\dagger}$ & $92.5 \% \pm 2.7 \%^{\dagger}$ & $81.7 \% \pm 5.6 \%^{*}$ & $81.9 \% \pm 7.1 \%^{* \times}$ & $83.6 \% \pm 7.4 \%^{* \times}$ & $87.4 \% \pm 4.0 \%^{\times}$ \\
\hline
\end{tabular}

\section{G. Performance Evaluation of the OSG using regional probabilities}

We performed additional tests to check the performance of the presented methods OSG-BB, OSG-BBEPI, and OSG-ALL. We compared these methods with manual segmentations from a second expert observer (observer 2) with a similar experience as observer 1, we compared the methods with another method proposed in the literature, and finally we tested the scan-rescan reproducibility of the methods. The following experiments include the six discarded cases with centerline errors as here we wanted to evaluate the entire pipeline. 


\section{Comparison with a second observer}

First, we compared the methods segmentation results to the manual segmentations from a second observer which were available in a subset of 27 arteries from 15 subjects. Segmented volumes were cropped as in the previous section. Not the complete bifurcation was evaluated as the second observer only segmented one of the branches. DSCs for wall, lumen, and complete vessel are listed in Table III. In 5 out of 6 comparisons, the wall overlap between the automatic method and the observer was significantly better than the overlap between the two observers. Additionally, scatter plots showing the wall volume correlation between OSG-BB and manual volumes are depicted in Figure 4(a). High correlations between wall volumes are observed.

TABLE III: DSC performance comparison between OSG-BB, OSG-BBEPI, OSG-ALL and the manually segmented volumes from two observers in a subset of 27 arteries for which two manual annotations are available. Values in the same row with the same symbol are not significantly different $(p>0.05)$.

OSG-BB Vs. Obs. $1 \quad$ OSG-BBEPI Vs. Obs. $1 \quad$ OSG-ALL Vs. Obs. $1 \quad$ OSG-BB Vs. Obs. 2 OSG-BBEPI Vs. Obs. 2 OSG-ALL Vs. Obs. 2 Obs. 1 Vs. Obs. 2

\begin{tabular}{|c|c|c|c|c|c|c|c|}
\hline Wall DSC & $73.8 \% \pm 6.7 \%^{\dagger}$ & $73.4 \% \pm 6.6 \%^{\dagger}$ & $74.7 \% \pm 4.8 \%^{\dagger}$ & $70.7 \% \pm 8.7 \%^{\dagger}$ & $69.9 \% \pm 9.2 \%^{\dagger \times}$ & $71.1 \% \pm 8.8 \%^{\dagger}$ & $65.1 \% \pm 8.9 \%^{\times}$ \\
\hline Lumen DSC & $88.5 \% \pm 4.8 \%^{\dagger}$ & $89.3 \% \pm 3.5 \%^{\dagger}$ & $89.5 \% \pm 3.2 \%^{\dagger}$ & $85.9 \% \pm 5.7 \%^{\dagger}$ & $85.9 \% \pm 4.9 \%^{\dagger}$ & $86.6 \% \pm 5.0 \%^{\dagger}$ & $81.9 \% \pm 5.1 \% \times$ \\
\hline Complete DSC & $93.2 \% \pm 2.7 \%^{\dagger}$ & $93.3 \% \pm 2.2 \%^{\dagger}$ & $93.5 \% \pm 1.9 \%^{\dagger}$ & $88.9 \% \pm 3.7 \%^{\times}$ & $89.3 \% \pm 3.6 \% \times$ & $89.1 \% \pm 3.9 \%^{\times}$ & $90.3 \% \pm 3.2 \%^{\times}$ \\
\hline
\end{tabular}

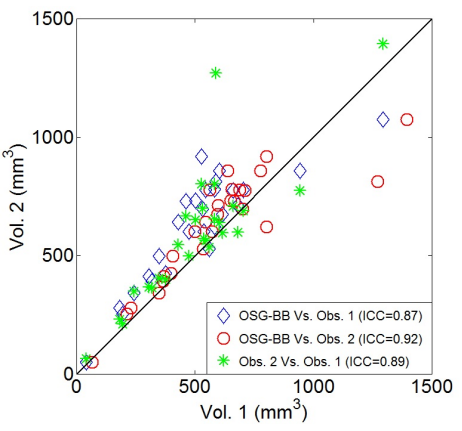

(a)

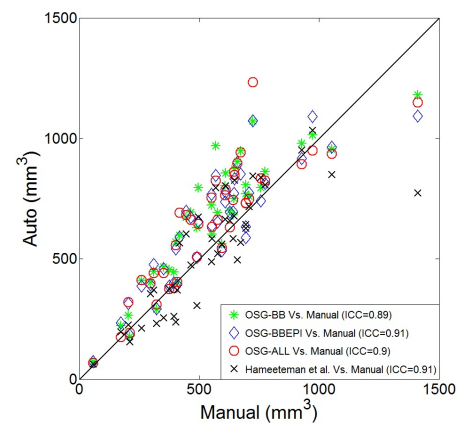

(b)

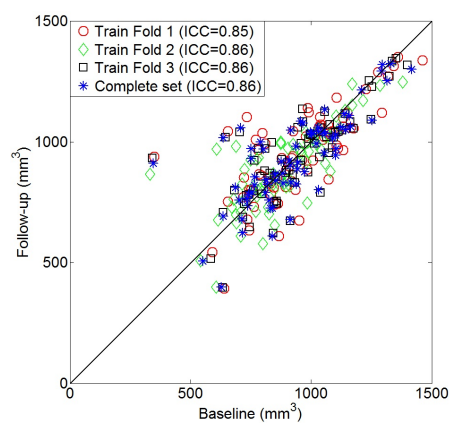

(c)

FIG. 4: Scatter plots (Vol. 2 Vs. Vol. 1) between: (a) the automatic and the two observer segmentations in a subset of 27 arteries for which two manual annotations are available, (b) OSG-BB, OSG-BBEPI, OSG-ALL and the method presented by Hameeteman et al. [6] and the manual segmentations from observer 1 using a subset of 41 arteries, and (c) follow-up wall volumes vs. Baseline wall volumes using a scan-rescan data set of 71 arteries, for the method OSG-BB trained on a complete data set of 49 arteries and its 3 training sets (see Section III B)

\section{Comparison with Hameeteman et al. [6]}

Additionally, we compared the presented methods to the best method presented by Hameeteman et al. [6], which is a method based on a cylindrical deformable surface model as in [26] but with a learning-based postprocessing. We used the publicly available dataset of [6] for this comparison, which consists of 41 arteries from 22 subjects which are a subset of the data presented in the rest of this paper. As the method proposed by Hameeteman et al. [6] cannot segment the complete bifurcation, only the common and internal carotid arteries were evaluated. DSCs values for the different methods are shown in Table IV. Significantly better results $(p<0.05)$ were obtained using the presented method for OSG-ALL. A scatter plot showing the wall volume correlations is depicted in Figure 4(b). Here, similar intra-class correlations were obtained. 
TABLE IV: DSC performance comparison between OSG-BB, OSG-BBEPI, OSG-ALL and the method presented by Hameeteman et al. [6] with respect to the manual segmented volumes from observer 1 using a subset of 41 arteries. Values in the same row with the same symbol are not significantly different $(p>0.05)$.

OSG-BB OSG-BBEPI OSG-ALL Hameeteman et al. [6]

\begin{tabular}{|c|c|c|c|c|}
\hline Wall DSC & $74.1 \% \pm 6.3 \%^{\dagger \times}$ & $73.7 \% \pm 6.6 \%^{\dagger \times}$ & $74.1 \% \pm 6.2 \%^{\dagger}$ & $67.0 \% \pm 13.4 \% \times$ \\
\hline Lumen DSC & $88.8 \% \pm 4.4 \%^{\dagger \times}$ & $89.0 \% \pm 3.5 \%^{\dagger \times}$ & $89.1 \% \pm 4.1 \%^{\dagger}$ & $86.6 \% \pm 7.7 \%^{\times}$ \\
\hline Complete DSC & $92.8 \% \pm 2.7 \%^{\dagger}$ & $93.0 \% \pm 2.4 \%^{\dagger}$ & $92.9 \% \pm 2.9 \%^{\dagger}$ & $89.3 \% \pm 6.8 \%^{\times}$ \\
\hline
\end{tabular}

3. Reproducibility analysis

Scan-rescan reproducibility was assessed on a different set of 71 arteries from 36 patients (one artery was discarded because the seed points were placed wrongly) who were imaged twice within a short time interval (15 \pm 9 days), so significant wall volume changes are not expected. For these experiments, we retrained and optimized the SVM and graph parameters for OSG-BB, OSG-BBEPI, OSG-ALL using all 30 subjects with manually segmented arteries annotated by observer 1 . The resulting parameters were: for OSG-BB: $\mathrm{C}=100, \gamma=0.01, p^{1}=100, p^{2}=500$, $\sigma=1.2$; for OSG-BBEPI: $\mathrm{C}=10, \gamma=0.01, p^{1}=0, p^{2}=500, \sigma=1.2$; and for OSG-ALL: $\mathrm{C}=1, \gamma=0.01, p^{1}=200$, $p^{2}=400, \sigma=1.2$. Additionally, to evaluate the effect of changes in the training data, we used the trained SVM and optimized parameters from each of the three training cross-validation sets composed of 20-21 subjects (see Table I). All segmented volumes were cropped from $18 \mathrm{~mm}$ below up to $2.7 \mathrm{~mm}$ above a manually indicated carotid bifurcation point in order to compare similar regions between baseline and follow-up. A scatter plot showing the correlations between vessel wall volumes at baseline and follow-up using the optimal parameters for each training set of OSG-BB is shown in Figure 4(c). Similar intra-class correlations are shown using each set of parameters, indicating good robustness of the method. Higher correlations were obtained using OSG-BB, with absolute volume differences (for the methods trained on the complete set) of $12 \% \pm 21 \%$ for OSG-BB, $14 \% \pm 24 \%$ for OSG-BBEPI, and $14 \% \pm 24 \%$ for OSG-ALL.

\section{DISCUSSION}

In this paper, we proposed a new segmentation method for coupled surfaces that maximizes the regional probabilities within each segmented region using a coupled OSG method. This method was applied to segment the carotid artery wall in MRI.

This method is especially useful for this application as the used graph structure allows the segmentation of high curvature surfaces at the carotid bifurcation. Similar methods for multi-region coupled surfaces segmentation as the one presented in Haeker et al. [15] and Sun et al. [17] are suitable to segment terrain-like and cylindrical surfaces but can not directly be applied to high curvature surfaces as the carotid artery.

Other segmentation methods that use regional information which are not based on OSG models [4, 13, 16] do not introduce an initialization shape prior in their model, which in our specific application proved to be useful. In addition, our regional information based on an SVM classifier showed very robust results for this application, while in the other methods only a limited set of regional features is considered. We argue that our method is suitable for any coupled multi-surface segmentation problem for which it is possible to obtain a coarse initial segmentation of any of the surfaces to segment.

We compared with a previously presented method for carotid wall segmentation [6], which already performed better than another previously published method [3]. We obtained similar wall volume correlations, and better and more robust DSC overlaps. Additionally, our method is more suitable for segmenting the bifurcation, since it incorporates this geometry into the method, while in Hameeteman et al.[6] it is necessary to combine two tubular segmentations. There are other methods to segment the artery wall, e.g. [4], which presented average lumen DSCs above 86\% and complete vessel DSCs above $87 \%$. These results are similar to the presented results by our method, however it is difficult to compare with these as the data is different. Additionally our method requires less user interaction compared to Ukwatta et al. [4].

Our results showed that an OSG approach combined with classifier-based regional probabilities improved wall 
segmentations over both an OSG method using only edge information as presented in Arias et al. [5], and a pure voxel classification-based method. Our explanation for this is that the method that only uses edge information tends to find the stronger edge in the image which may not be the artery edge, and that the classifier alone does not have any geometrical constraints which leads to more irregular surfaces and leakage into other structures that resemble the arterial wall. However, OSG-Edge resulted in better lumen segmentations, which could be explained by the good lumen contrast so surface information represented by only directional derivatives are more descriptive of the lumen border than using regional information. A combination of both surface and regional information similar to Haeker et al. [15] and Sun et al. [17] could be used to avoid the performance reduction in the lumen segmentation.

In our experiments combining information from multiple image sequences did not have a big impact. Although slightly better voxel-wise classification results were obtained when including more image sequences, inaccuracies in the RPMs based on only BB images were easily corrected using the OSG. However, a feature selection method to see which images and features are more important could be interesting to perform in the future.

The OSG method is not very sensitive to changes in the model parameters, as similar performances were obtained with different parameters in the reproducibility analysis. However, if different data is used (for instance images from a different scanner or using a different MRI protocol), re-training the SVM classifiers is likely to be necessary. On a different application OSG parameters naturally require retraining as well.

Although we obtained good overlap with manual segmentations, the obtained surfaces were not always smooth, as during the training of the method, we were aiming to get the highest wall overlapping without considering the smoothness of the segmented surfaces. A parameter training also targeting smoother results could help to get more visually appealing segmentations.

Using the proposed method we obtained better wall DSC compared to two different observers. We noticed a wall volume bias between observers, indicating that one of the observers were was over-segmenting the wall compared to the other observer. The results of our method agree better with Observer 1, which is the observer whose annotations were used for training. However, DSCs between results of our method and both observers were larger than between observers, which may indicate that the automatic segmentation contours are closer to the true artery borders.

Reproducibility experiments showed good correlations between scan and re-scan segmented volumes. This correlation is similar to the correlation obtained between observers, which shows that the method is highly reproducible. The observed scan and re-scan segmented volume differences $(<14 \%$ volume change) are significantly lower than what has been reported in clinical time intervals ( $59 \%$ volume change in 18.9 months interval, see [28]), which suggest that this method can be reliably applied in longitudinal studies. While we evaluated the method in relatively healthy arteries in a population study, we expect this method could be used in more diseased patients, as we observed fairly good segmentations in the diseased sections. Additionally, even though our dataset is larger than other studies datasets $([4,6])$, this is still not very large, and therefore we handled that by doing a cross-validation.

\section{CONCLUSION}

In conclusion, we formulated a novel cost function to maximize regional probabilities using the coupled OSG model presented in Petersen et al. [10]. With our proposed method the maximum sum of enclosed class-conditional probabilities in each region is achieved while ensuring smooth surfaces and topologically correct segmentations. We applied this method to segment the carotid artery wall in MRI. The method outperformed a voxel-wise segmentation approach using the regional probability maps only, as well as an OSG segmentation using only edge information, and a recently published method. Obtained automatic segmentation results were of similar quality as manual segmentations by experienced observers and showed good scan-rescan reproducibility.

\section{Acknowledgements}

This research was financially supported by the Netherlands Organization for Scientific Research (NWO) and the Danish Council for Independent Research. 
[1] Sitzer M., Muller W., Siebler M., et al. Plaque ulceration and lumen thrombus are the main sources of cerebral microemboli in high-grade internal carotid artery stenosis Stroke. 1995;26:1231-1233.

[2] Hameeteman K., Zuluaga M.A., Freiman M., et al. Evaluation framework for carotid bifurcation lumen segmentation and stenosis grading Medical Image Analysis. 2011;15:477-488.

[3] Klooster Ronald, Koning Patrick J.H., Dehnavi Reza Alizadeh, et al. Automatic lumen and outer wall segmentation of the carotid artery using deformable three-dimensional models in MR angiography and vessel wall images Journal of Magnetic Resonance Imaging. 2012;35:156-165.

[4] Ukwatta E., Yuan Jing, Rajchl M., Qiu Wu, Tessier D., Fenster A.. 3-D Carotid Multi-Region MRI Segmentation by Globally Optimal Evolution of Coupled Surfaces Medical Imaging, IEEE Transactions on. 2013;32:770-785.

[5] Arias-Lorza A. M., Petersen J., Engelen A., et al. Carotid Artery Wall Segmentation in Multispectral MRI by Coupled Optimal Surface Graph Cuts IEEE Transactions on Medical Imaging. 2016;35:901-911.

[6] Hameeteman K., Klooster R., Selwaness M, et al. Carotid wall volume quantification from magnetic resonance images using deformable model fitting and learning-based correction of systematic errors Physics in Medicine and Biology. $2013 ; 58$.

[7] Freiman Moti, Broide Noah, Natanzon Miriam, et al. Vessels-Cut: a graph based approach to patient-specific carotid arteries modeling in Proceedings of the 2009 international conference on Modelling the Physiological Human3DPH'09(Berlin, Heidelberg):1-12Springer-Verlag 2009.

[8] Bauer Christian, Pock Thomas, Sorantin Erich, Bischof Horst, Beichel Reinhard. Segmentation of interwoven 3d tubular tree structures utilizing shape priors and graph cuts Medical Image Analysis. 2009.

[9] Xu X., Niemeijer M., Song Q., Garvin M.K., Reinhardt J.M., Abramoff M.D.. Retinal vessel width measurements based on a graph-theoretic method in Biomedical Imaging: From Nano to Macro, 2011 IEEE International Symposium on:641-644 2011.

[10] Petersen Jens, Nielsen Mads, Lo Pechin, et al. Optimal surface segmentation using flow lines to quantify airway abnormalities in chronic obstructive pulmonary disease Medical Image Analysis. 2014;18:531-541.

[11] Boykov Y., Kolmogorov V.. An experimental comparison of min-cut/max-flow algorithms for energy minimization in vision IEEE Transactions on Pattern Analysis and Machine Intelligence. 2004;26:1124-1137.

[12] Wu Xiaodong, Chen Danny. Optimal Net Surface Problems with Applications in Automata, Languages and Programming (Widmayer Peter, Eidenbenz Stephan, Triguero Francisco, Morales Rafael, Conejo Ricardo, Hennessy Matthew. , eds.);2380 of Lecture Notes in Computer Science:775-775Springer Berlin / Heidelberg 2002.

[13] Delong A., Boykov Y.. Globally optimal segmentation of multi-region objects in Computer Vision, 2009 IEEE 12th International Conference on:285-292 2009.

[14] Wu Xiaodong, Dou Xin, Wahle A., Sonka M.. Region Detection by Minimizing Intraclass Variance With Geometric Constraints, Global Optimality, and Efficient Approximation Medical Imaging, IEEE Transactions on. 2011;30:814-827.

[15] Haeker M., Wu X., Abramoff M., Kardon R., Sonka M.. Incorporation of regional information in optimal 3-D graph search with application for intraretinal layer segmentation of optical coherence tomography images Inf Process Med Imaging. 2007;20:607-618.

[16] Lecellier François, Jehan-Besson Stéphanie, Fadili J. Statistical region-based active contours for segmentation: an overview IRBM. 2014;35:3-10.

[17] Sun Shanhui, Sonka Milan, Beichel Reinhard R. Graph-based IVUS segmentation with efficient computer-aided refinement IEEE transactions on medical imaging. 2013;32:1536-1549.

[18] Li Kang, Wu Xiaodong, Chen Danny Z., Sonka Milan. Optimal surface segmentation in volumetric images - a graphtheoretic approach IEEE Transactions on Pattern Analysis and Machine Intelligence. 2006;28:119-134.

[19] Liu Xiaomin, Chen Danny Z, Tawhai Merryn H, Wu Xiaodong, Hoffman Eric A, Sonka Milan. Optimal graph search based segmentation of airway tree double surfaces across bifurcations IEEE transactions on medical imaging. 2013;32:493-510.

[20] Ishikawa H.. Exact optimization for Markov random fields with convex priors Pattern Analysis and Machine Intelligence, IEEE Transactions on. 2003;25:1333-1336.

[21] Bouwhuijsen Quirijn J.A., Vernooij Meike W., Hofman Albert, Krestin Gabriel P., Lugt Aad, Witteman Jacqueline C.M.. Determinants of magnetic resonance imaging detected carotid plaque components: the Rotterdam Study European Heart Journal. 2011.

[22] Chang Chih-Chung, Lin Chih-Jen. LIBSVM: A library for support vector machines ACM Transactions on Intelligent Systems and Technology. 2011;2:27:1-27:27.

[23] Wu Ting-Fan, Lin Chih-Jen, Weng Ruby C. Probability estimates for multi-class classification by pairwise coupling Journal of Machine Learning Research. 2004;5:4.

[24] Tang H., Walsum T., Onkelen R. S., et al. Semiautomatic Carotid Lumen Segmentation for Quantification of Lumen Geometry in Multispectral MRI Medical Image Analysis. 2012;16:1202-1215.

[25] Tustison N.J., Avants B.B., Cook P.A., et al. N4ITK: Improved N3 bias correction IEEE Transactions on Medical Imaging. 2010;29(6):1310 - 1320 .

[26] Klooster R., Staring M., Klein S., et al. Automated registration of multispectral MR vessel wall images of the carotid 
artery Medical Physics. 2013;40:121904-1 - 121904-12.

[27] Silvestrini Mauro, Rizzato Barbara, Placidi Fabio, Baruffaldi Roberto, Bianconi Alberto, Diomedi Marina. Carotid Artery Wall Thickness in Patients With Obstructive Sleep Apnea Syndrome Stroke. 2002;33:1782-1785.

[28] Schminke U., Motsch L., Griewing B., Gaull M., Kessler C.. Three-dimensional power-mode ultrasound for quantification of the progression of carotid artery atherosclerosis J. Neurol.. 2000;247:106-111. 